\title{
The Effects on Technologist Occupational Exposure in PET/CT Departments When Working with Students at Various Levels of Supervision
}

\author{
Jacob Farkas, Michael Martin, Cybil Nielsen, and S. Gregory Jennings \\ Indiana University School of Medicine, Indianapolis, Indiana
}

\begin{abstract}
The purpose of this study was to evaluate the effect that the presence of a student in the PET/CT department has on the technologist's occupational radiation exposure and whether this effect is influenced by the type of supervision performed. Methods: This was a retrospective, institutional review boardapproved study that collected data from 2 PET/CT departments. Dosimetry reports, correlated with the clinical schedules of the students, were normalized for workflow (amount of radioactivity), the number of technologists, and the number of monitored days in the department. A 2-sample $t$ test assuming unequal variance with an a of 0.05 was used to compare doses between with-student and without-student groups and between direct-supervision and indirect-supervision groups. Results: The study consisted of a dataset of 42 dosimetry reports, 19 with students and 23 without students. When comparing withstudent and without-student groups, the total $(n=42)$ extremity dose had a $P$ value of 0.012 with a mean of $0.0011665 \mu \mathrm{Sv} /$ $\mathrm{MBq} /$ technologist/d; all other dose comparisons between groups were greater than $0.05(P>0.05)$. For indirect supervision $(n=21)$, the extremity-dose $P$ value was 0.298 . The other dose $P$ values were all less than 0.05 . For direct supervision $(n=$ 21 ), the dose $P$ values were all greater than 0.05 . There was a trend toward decreasing exposure of technologists when students were in the department. Conclusion: Extremity dose decreases when students are present. There is a trend toward decreasing dose with indirect supervision.
\end{abstract}

Key Words: PET; PET/CT; radiation safety; exposure; students; supervision

J Nucl Med Technol 2020; 48:214-217

DOI: 10.2967/jnmt.119.241398

$\mathbf{N}$ uclear medicine technologists are continuously monitored for radiation exposure throughout their careers. The Nuclear Regulatory Commission has set occupational exposure limits to ensure that the levels to which technologists are exposed are below the levels at which effects are

Received Dec. 23, 2019; revision accepted Mar. 28, 2020.

For correspondence or reprints contact: Jacob Farkas, Indiana University School of Medicine, 1120 W. Michigan St., Indianapolis, IN 46202.

E-mail: jafarkas@iu.edu

Published online Apr. 20, 2020.

COPYRIGHT (c) 2020 by the Society of Nuclear Medicine and Molecular Imaging. observed. Radiation monitoring badges should be worn at the correct locations on the body; otherwise, readings may be skewed (1). Though these limits are rarely reached, the radiation exposure in the workplace itself may raise concerns for nuclear medicine technologists.

In the PET/CT department, technologists tend to alternate with one another regarding patient preparation in order to reduce their exposure. For example, if the department has 3 technologists, 1 technologist would inject every third patient. When a student is present in the PET/CT department, the student's presence can either be used for benefit or be seen as a potential risk of more exposure.

Radiopharmaceuticals used in PET require specialized personnel, facilities, and equipment, primarily because of the relatively short physical half-lives of the radionuclides and the relatively high exposure of the technologist to radiation (2). The 3 main principles to be mindful of when dealing with radiation exposure are time, distance, and shielding. By having a student complete marginal tasks, the technologist may be able to maximize adherence to these principles.

Having a student inject the radioactive tracer may significantly reduce the technologist's radiation exposure. A 2005 study on reducing the radiation exposure of technologists showed that the bulk of a technologist's exposure in the PET/CT department comes from injecting the dose (3), largely because the radioactive material is no longer shielded once put into the body (4). Other normal student duties include accompanying patients from the injection rooms, positioning patients on the table, asking patients to get off the table, and walking patients out of the clinic. These duties help the technologist increase distance from and reduce time spent with the radioactive patient.

There are 2 main teaching strategies in the clinical setting, indirect supervision and direct supervision. According to the Joint Review Committee on Educational Programs in Nuclear Medicine Technology Standards, "Direct supervision of students is required at clinical affiliates until competence is demonstrated, after which time supervision may be indirect. Direct supervision requires the clinical instructor to be physically present with the student. Indirect supervision requires the clinical instructor to be within the facility and 


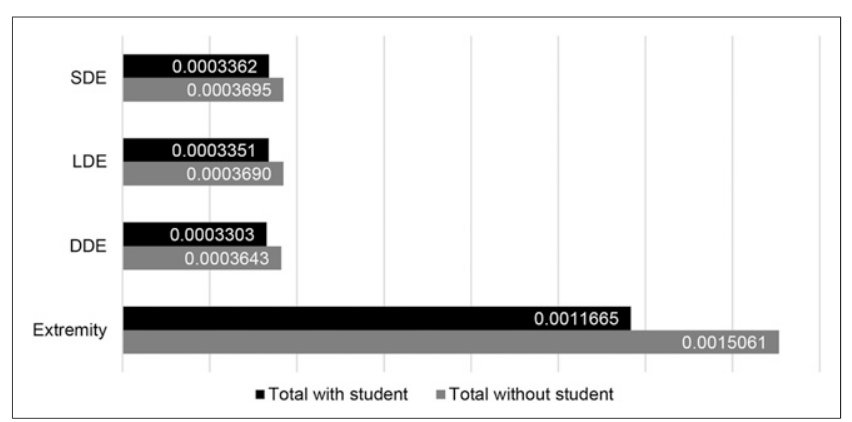

FIGURE 1. $P$ values for all departments: with vs. without students. $\mathrm{DDE}=$ deep-dose equivalent; LDE = lens-dose equivalent; $\mathrm{SDE}=$ shallow-dose equivalent.

immediately available to provide direct supervision" (5). Students should be supervised by the technologists they are working with, but the level of supervision is not prescribed. Direct supervision fulfills this requirement but does not allow the technologist to use the radiation protection principles (5).

The purpose of this study was to evaluate the effect that the presence of a student in the PET/CT department has on the technologist's occupational radiation exposure and whether this effect is influenced by the type of supervision performed.

\section{MATERIALS AND METHODS}

Institutional review board approval was received, and Health Insurance Portability and Accountability Act guidelines were followed. This was a retrospective study using data collected from 2 PET/CT departments. The 2 departments were selected because both had full-time PET/CT technologists who worked only in the PET/CT department and did so consistently over the 23-mo study period (June 2017 to April 2019). The data collected included monthly occupational dosimetry reports and the total monthly prescribed radioactivity. The dosimetry reports contained data on the lens-dose equivalent, deep-dose equivalent, shallow-dose equivalent, and extremity dose. The number of technologists in each department was recorded, as well as the number of days monitored for each monthly exposure report. Each monthly data point was separated into 2 categories: with a student (student days in department $\geq 1$ ) and without a student (student days in department $=0$ ). They were further divided into 2 supervision strategies: direct supervision and indirect supervision. Departments were categorized as indirect supervision if students were allowed to be alone with the patient during tasks such as injection, patient education and history, dose administration, scanning, and processing. Departments were categorized as direct supervision if the technologist was physically present with the student during these tasks.

The occupational exposure $(\mu \mathrm{Sv})$ was then normalized for patient workload ( $\mathrm{MBq}$ prescribed), number of technologists working in the department, and days monitored. The normalized data were presented in base units of $\mu \mathrm{Sv} / \mathrm{MBq} / \mathrm{technologist} / \mathrm{d}$. The datasets for exposure with and without students were analyzed through a 2 -sample $t$ test assuming unequal variance with an $\alpha$ of 0.05 . The data subsets for direct supervision and indirect supervision were analyzed in the same way.

\section{RESULTS}

In total, 42 dosimetry reports were collected: 19 for the with-student group and 23 for the without-student group. When the 2 groups were compared, the extremity dose had a $P$ value of 0.012 with a mean of $0.0011665 \mu \mathrm{Sv} / \mathrm{MBq} /$ technologist/d. The deep-dose equivalent, lens-dose equivalent, and shallow-dose equivalent $P$ values were greater than 0.05 ; though not significantly different, all 3 had lower means for the with-student group (Fig. 1).

For direct supervision $(n=21)$, the extremity dose, deep-dose equivalent, lens-dose equivalent, and shallow-dose equivalent $P$ values were greater than 0.05 , but with means that were lower for the with-student group (Table 1). For indirect supervision, the extremity-dose $P$ value was greater than 0.05 . The deep-dose equivalent, lens-dose equivalent, and shallow-dose equivalent $P$ values were less than 0.05. All categories showed lower mean exposure for the with-student group than the without-student group (Table 2; Figs. 2 and 3).

\section{DISCUSSION}

Though the $P$ values did not show a statistically significant difference in most cases, the overall trend of the means showed a reduced exposure when a student was present in the PET/CT department. There was no significant difference in exposure between departments using direct supervision and departments using indirect supervision; both showed a significant dose reduction to the technologists. The means within the supervision subsets themselves

TABLE 1

Percentage Reduction in Exposure for Indirect-Supervision Departments and Direct-Supervision Departments Depending on Whether Students Were Present

\begin{tabular}{lcccc}
\hline \multicolumn{1}{c}{ Parameter } & Extremity & Deep-dose equivalent & Lens-dose equivalent & Shallow-dose equivalent \\
\hline $\begin{array}{l}\text { Indirect-supervision departments } \\
\text { With students }\end{array}$ & 0.0013867 & 0.0002642 & 0.0002672 & 0.0002700 \\
$\quad$ Without students & 0.0016225 & 0.0003408 & 0.0003452 & 0.0003459 \\
$\quad \begin{array}{l}\text { Percent reduction in exposure } \\
\text { Direct-supervision departments }\end{array}$ & $14.5 \%$ & $22.5 \%$ & $22.6 \%$ & $22.0 \%$ \\
$\quad$ & 0.0010380 & 0.0003689 & 0.0003748 & 0.0003748 \\
$\quad$ With students & 0.0013251 & 0.0004007 & 0.0004061 & 0.0004061 \\
$\quad$ Without students & $21.7 \%$ & $7.9 \%$ & $7.7 \%$ & $7.7 \%$ \\
\hline Percent reduction in exposure & & & & \\
\hline
\end{tabular}


TABLE 2

$P$ Values Comparing When Students Are Present and When They Are Not, by Department Type

\begin{tabular}{lcccc}
\hline \multicolumn{1}{c}{ Department } & Extremity & Deep-dose equivalent & Lens-dose equivalent & Shallow-dose equivalent \\
\hline All & $0.01206^{\star}$ & 0.14676 & 0.15299 & 0.15722 \\
Direct supervision & 0.09177 & 0.20746 & 0.21274 & 0.21274 \\
Indirect supervision & 0.29835 & $0.03316^{\star}$ & $0.03536^{\star}$ & $0.04091^{*}$
\end{tabular}

${ }^{*}$ Statistically significant difference $(P<0.05)$.

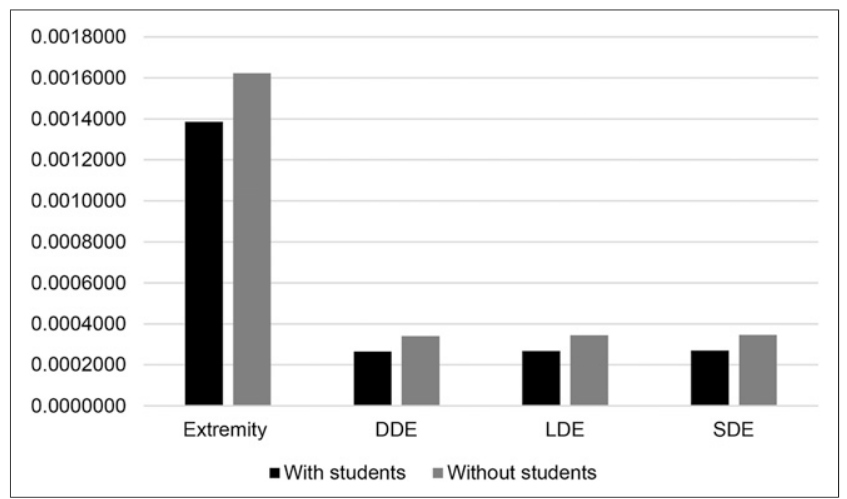

FIGURE 2. $P$ values for indirect-supervision departments only: with vs. without students. DDE $=$ deep-dose equivalent; $L D E=$ lens-dose equivalent; SDE = shallow-dose equivalent.

still showed a reduction in dose with a student in the department. These results could be related to the amount of work the student does for the technologist.

The radionuclide energies used in the PET/CT department are considerably higher and result in a higher wholebody dose than the radionuclides used in the general nuclear medicine department (6). For this reason, students may not be introduced to PET/CT in their early stages of training. In some nuclear medicine technology programs, students rotate through general nuclear medicine first, to obtain skills such as injection techniques and to practice radiation protection around lower-energy radionuclides, before going into the PET/CT department.

The Joint Review Committee on Educational Programs in Nuclear Medicine Technology suggests that clinical sites should operate under competency-based clinical education guidelines (5). It is important that technologists follow these guidelines for supervision because, as our results indicate, the level of supervision may affect the amount of radiation the technologist receives. Technologists who work at an educational facility should be instructed on how to effectively train a student and should use these guidelines in order to reduce their radiation exposure.

Because of the higher radiation risks, students may develop their injection techniques and radiation protection skills in the general department before continuing to PET/ CT. Before students are introduced to the PET/CT depart-

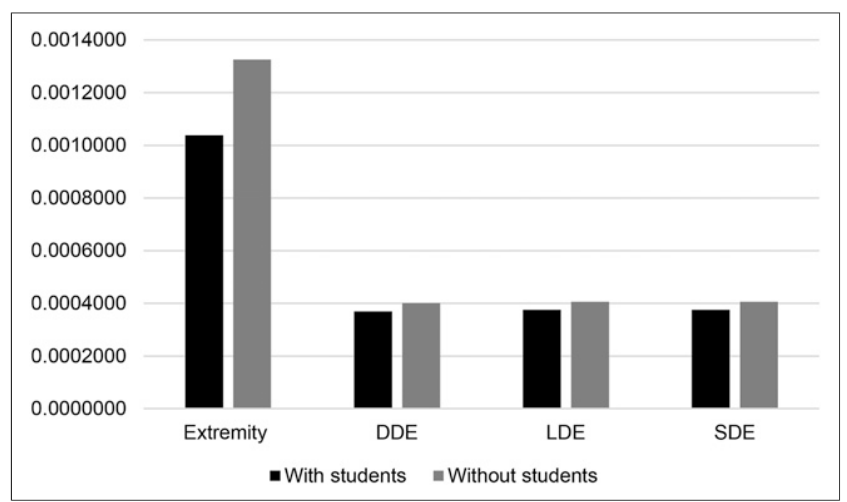

FIGURE 3. $P$ values for direct-supervision departments only: with vs. without students. $\mathrm{DDE}=$ deep-dose equivalent; $\mathrm{LDE}=$ lens-dose equivalent; SDE = shallow-dose equivalent.

ment, they should have achieved a substantial number of competent skills. To take full advantage of a student's presence in the department, a technologist should take the time to find out the skills of the student and use indirect supervision for tasks requiring those skills.

There were limitations to this study. The number of days the student was in the department was not gathered. That information could lead to a further understanding of how much a student can reduce a technologist's exposure. It was assumed that technologists wore their badges each day and wore them correctly. It was also assumed that the PET/CT technologists did not have any other technologist working part-time during the 23-mo study period. A further limitation was that technologists might alter their injection techniques when students are present, perhaps opting for a more conventional technique. A nonconventional technique might lead to higher or lower radiation exposure. Since the study was retrospective, the injection techniques could not be regulated. A further evaluation of exposure should be expanded to general nuclear medicine departments as well, along with departments that have more than 1 student present.

\section{CONCLUSION}

Extremity dose decreases when students are present. There is a trend toward decreasing dose with indirect supervision. 


\section{DISCLOSURE}

No potential conflict of interest relevant to this article was reported.

\section{REFERENCES}

1. Salesses F, Perez P, Maillard AE, Blanchard J, Mallard S, Bordenave L. Effect of dosimeter's position on occupational radiation extremity dose measurement for nuclear medicine workers during ${ }^{18}$ F-FDG preparation for PET/CT. EJNMMI Phys. 2016;3:16.

2. Callahan RJ, Chilton HM, Ponto JA, Swanson DP, Royal HD, Bruce AD. Procedure guideline for the use of radiopharmaceuticals 4.0. J Nucl Med Technol. 2007;35:272-275
3. Roberts FO, Gunawardana DH, Pathmaraj K, et al. Radiation dose to PET technologists and strategies to lower occupational exposure. J Nucl Med Technol. 2005;33:44-47.

4. Peet DJ, Morton R, Hussein M, Alsafi K, Spyrou N. Radiation protection in fixed PET/CT facilities: design and operation. Br J Radiol. 2012;85:643-646.

5. Accreditation standards for nuclear medicine technologist education. Joint Review Committee for Education Programs in Nuclear Medicine Technology website. https://www.jrcnmt.org/sites/jrcnmt2/uploads/documents/Accred_ Policy_Documents/Standards_Nov2019rev.pdf. Published 2017. Accessed June $17,2020$.

6. Guillet B, Quentin P, Waultier S, Bourrelly M, Pisano P, Mundler O. Technologist radiation exposure in routine clinical practice with ${ }^{18}$ F-FDG PET. $\mathrm{J}$ Nucl Med Technol. 2005;33:175-179. 